\section{Chalcogenide glass thin films through inverted deposition and high velocity spinning}

\author{
A.K. Mairaj, R.J. Curry and D.W. Hewak
}

The fabrication of optical chalcogenide glass thin films through inverted deposition and high velocity spinning is presented. Good quality films up to $275 \mu \mathrm{m}$ thick of this infrared transmitting material, with propagation loss of $0.3 \mathrm{~dB} \mathrm{~cm}^{-1}$ at $1.064 \mu \mathrm{m}$, were engineered using this technique.

Introduction: The increasing sophistication of the industries requiring optical thin films from unconventional materials calls for alternative fabrication methods. Thin film for use in broadband and narrowband filtering applications, including dense wavelength division multiplexing (DWDM), optical amplification, switching, modulation of light signals, grating based devices, infrared light transmission, focusing, and as antireflection coatings are just some of the target areas, although other technological sectors including displays, aerospace, biomedical and defence have requirements. For the infrared (IR) transmitting family of glasses, in particular chalcogenides, several techniques for the production of thin films have been reported $[1,2]$. Chalcogenide glasses (ChGs) contain elements from group VI of the periodic table as glass-forming anions and are generally opaque in the visible, with the exception of some sulphide glasses. The transmission window for ChGs extends from $1 \mu \mathrm{m}$ to longer infrared wavelengths compared with oxide and fluoride glasses. The high-refractive index, enhanced mid-IR transmission in ChGs with reduced maximum phonon energy and ability to exhibit localised reversible or irreversible material changes when irradiated with light suggest these glasses are indeed interesting candidates for thin film development [3]. In this Letter we report the first steps in realising amorphous thin films in a $\mathrm{ChG}$ (gallium lanthanum sulphide, $\mathrm{Ga}: \mathrm{La}: \mathrm{S})$ utilising the inverted deposition of the molten phase combined with high velocity spinning.

Experimental procedure and results: The Ga:La:S family of glasses have optical transparency between 0.5 and $10 \mu \mathrm{m}$ (at $50 \%$ transmission through $1 \mathrm{~mm})$, a high glass transition temperature $\left(T_{g}\right)$ of $580^{\circ} \mathrm{C}$, they can be easily doped with rare earth, transition and precious metals (e.g. silver and platinum) and exhibit photosensitive properties. Fabrication of the substrates used in the experiments, with a typical molar composition $72.5 \mathrm{Ga}_{2} \mathrm{~S}_{3}: 21.5 \mathrm{La}_{2} \mathrm{~S}_{3}: 6 \mathrm{La}_{2} \mathrm{O}_{3}$, was carried out from prepared batches of powder precursors processed within our laboratories with a purity exceeding $99.999 \%$. These glass precursors were loaded into a vitreous carbon crucible while in a controlled nitrogen atmosphere. The precursors are non-volatile at the glass melting temperature $\left(1150^{\circ} \mathrm{C}\right.$ for up to $\left.24 \mathrm{~h}\right)$ and were heated in an open (argon purged) atmosphere, after which the melt was quenched and annealed. Each substrate shaped as a disc had a diameter and thickness of $29 \times 2 \mathrm{~mm}$, respectively, and was optically polished on the top and bottom face. An optically flat polish on the material deposition surface is essential as this enhances interfacial uniformity between the deposited film and substrate. The deposited glass had molar composition $70 \mathrm{Ga}_{2} \mathrm{~S}_{3}: 24 \mathrm{La}_{2} \mathrm{~S}_{3}: 6 \mathrm{La}_{2} \mathrm{O}_{3}$ with increased material polarisability providing $+\Delta n$ of 0.005 over the refractive index of the substrate glass. To function effectively as an optical waveguide, it is crucial that the deposited film is amorphous. Therefore, the separation of the peak $\left(T_{p}\right)$ and the onset of crystallisation temperature $\left(T_{x}\right)$ is required to be broad $\left(>25^{\circ} \mathrm{C}\right)$. A narrow separation $\left(<25^{\circ} \mathrm{C}\right)$ suggests a well-defined crystalline event which will have structural prevalence over any amorphous lattice configuration. However, careful optimisation of glass former, modifier and stabiliser content can result in significant suppression of crystal phases and favour the formation of an amorphous structure within the cooling thin film. These temperature events were characterised utilising a calibrated differential thermal analyser (Perkin Elmer DTA 7). A measured value of $52^{\circ} \mathrm{C}$ for the $T_{p}-T_{x}$ figure and an observed weak exothermic crystalline event $\left(T_{p}\right)$ for the substrate glass suggest sufficient resistance to crystallisation. The deposited glass composition had a measured $T_{p}-T_{x}$ value of $31^{\circ} \mathrm{C}$ in addition to a weak exothermic crystalline event (although more prominent than the substrate glass) and was considered to be suitably crystallisation resistant. The thermal expansion coefficients for the Ga:La:S glass substrate and deposited film were measured, utilising a calibrated thermal mechanical analyser (Perkin Elmer TMA 7), to be within $\pm 0.2 \times 10^{-6}{ }^{\circ} \mathrm{C}^{-1}$ of each other. Disparate thermal expansion coefficients between the substrate and deposited film can result in structural damage during cooling to ambient conditions.

The fabrication of amorphous thin films of Ga:La:S glass involved three stages, as shown in Fig. 1. The first stage involves the deposition of the coating fluid (molten glass) onto the substrate surface which is held in place by a boron nitride vacuum chuck. A crucible containing molten glass $\left(\sim 1250^{\circ} \mathrm{C}\right)$ makes slight contact with the substrate surface. The preheated Ga:La:S glass substrate $\left(\sim 540^{\circ} \mathrm{C}\right)$ is dipped approximately $1 \mathrm{~mm}$ into the molten Ga:La:S glass. It is vital that the substrate surface is completely wet with molten glass during this stage. Normally a substantial excess of coating fluid is deposited compared with the final film thickness. During the second stage the substrate is withdrawn, within $200 \mathrm{~ms}$ after contact, from the molten glass surface but still positioned within the walls of the crucible. The excess molten deposited glass on the substrate surface forms a meniscus as it drips back into the crucible. Positioning the substrate as such is a precautionary stage as any molten glass expelled from the substrate surface during the third stage (spin cycle) is collected within the crucible. The third stage is typically characterised by the expulsion of deposited fluid from the substrate surface through a rotational motion. The substrate is accelerated to a preset angular velocity for a specified duration and when spinning at a constant rate viscous forces dominate fluid thinning. The crucible is fully withdrawn from the substrate $2 \mathrm{~s}$ after the spin cycle has started. This reduces the effects of thermal radiation, emanating from the hot crucible and molten glass, affecting fluid flow and retarding the quench of the deposited film. The deposited molten film thins out and cools until the viscosity is too high for further flow to occur. Following the third stage, the substrate and deposited amorphous film are annealed at $540^{\circ} \mathrm{C}$ for $1 \mathrm{~h}$ and then slowly cooled, at $1^{\circ} \mathrm{C} \mathrm{min}^{-1}$, to an ambient temperature.

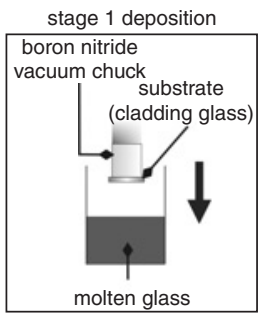

stage 2 withdrawal

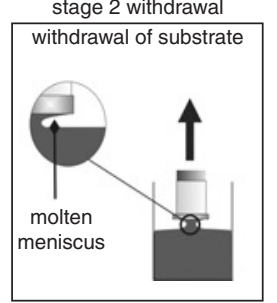

stage 2 withdrawal

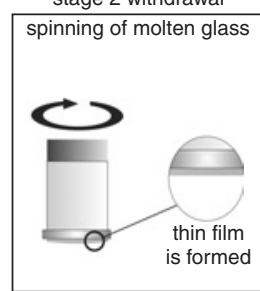

Fig. 1 Three-step process for inverted deposition spin coating of thin film glass waveguides

The sensitivity of a thin film to the environment during fabrication has some bearing on its quality. A critical variable is humidity and changes will vary quality of the deposited film. Deformation can manifest itself as film roughness, micro cracking and exaggerated formation of striations. In this work, experiments were carried out entirely in air, although it is desirable to maintain a self-contained inert processing environment. In Fig. 2 a micrograph obtained using an LEO 430 scanning electron microscope (SEM) shows a Ga:La:S glass substrate with a $275 \mu \mathrm{m}$ amorphous deposited higher refractive index Ga:La:S film. The film quality is observed to be excellent without the formation of bubbles, cracks or similar imperfections. The deposition condition for the amorphous film was spin velocity of $2500 \mathrm{rpm}$ for $10 \mathrm{~s}$ with surface uniformity variation $<0.5 \mu \mathrm{m}$ across $25 \mathrm{~mm}$ (profiled with a Tencor atomic force microscope). Significantly, crystallisation was observed neither in the deposited layer nor in the distinct interface between the deposited film and substrate. The film thickness as shown in Fig. 2 was reduced to $80 \mu \mathrm{m}$ (surface uniformity variation $<0.5 \mu \mathrm{m}$ across $25 \mathrm{~mm}$ ) using a spin velocity of $3500 \mathrm{rpm}$ for $15 \mathrm{~s}$. The absence of any micro and macro cracking indicates that $\alpha$ for the studied glasses are well matched. However, a tolerance of up to $\alpha= \pm 1.5 \times 10^{-6}{ }^{\circ} \mathrm{C}^{-1}$ is expected, thus allowing use of suitable commercially available substrates. The stoichiometric similarity between the deposited film and bulk glass was verified with an energy dispersive X-ray microscope (EDAX) and the measurements revealed compositional accuracy within $\pm 5 \%$. The propagation loss at $1.064 \mu \mathrm{m}$ for the film shown in Fig. 2 
was $0.3 \mathrm{~dB} \mathrm{~cm}^{-1}$ over a $15 \mathrm{~mm}$ path length, indicating good transmission and low scattering requirements for many applications.

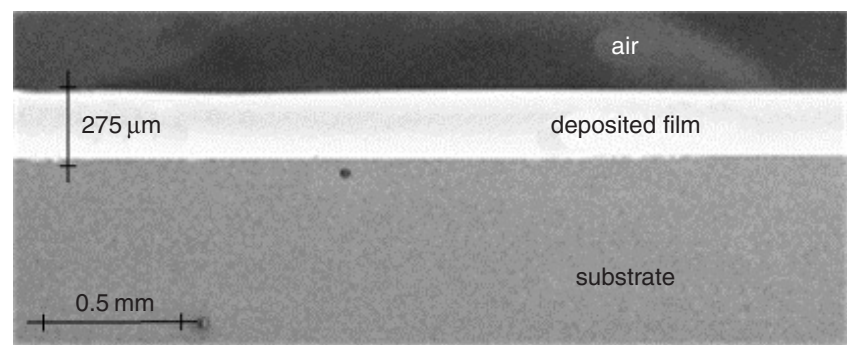

Fig. 2 Micrograph (SEM) of spun $275 \mu \mathrm{m}$ Ga:La:S glassy film having high uniformity and interfacial quality with substrate

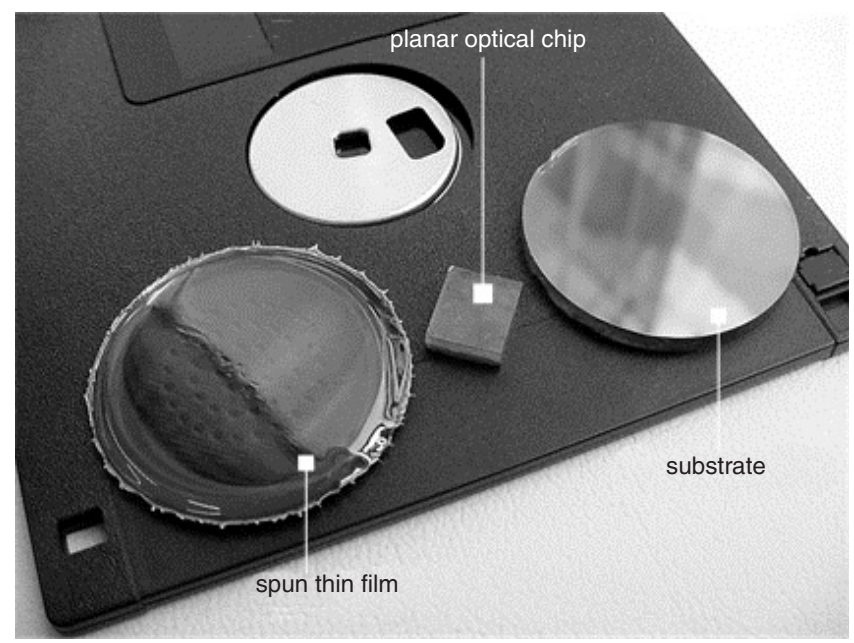

Fig. 3 (from left to right) Spun thin-film, planar optical chip and clad glass substrate from Ga:La:S glass system
Conclusions: We have demonstrated that a modified version of the conventional spin coating technique can be applied to a multicomponent chalcogenide glass to successfully produce uniform films with a thickness of a few hundred microns. In Fig. 3, a spun thin film, planar optical chip and glass substrate from the Ga:La:S glass system are shown. The planar optical chip has been cut from the spun thin film and can be scaled in size to suit an application. In demonstrating this technique we have developed a chalcogenide glass that can be quenched in air without oxygenation or devitrification occurring. As such, we believe that this technique can be developed further to deposit thin films of this glass for a variety of industrial applications.

Acknowledgments: The authors acknowledge contributions from D. Harwood. This work was supported by EPSRC grant GR/R941 $21 / 01$.

(C) IEE 2004

4 February 2004

Electronics Letters online no: 20040307

doi: 10.1049/el:20040307

A.K. Mairaj, R.J. Curry and D.W. Hewak (Optoelectronics Research Centre, University of Southampton, Southampton SO17 1BJ, United Kingdom)

\section{References}

1 Gmachl, C., Hwang, H.Y., Paiella, R., Sivco, D.L., Baillargeon, J.N., Capasso, F., and Cho, A.Y.: 'Quantum cascade lasers with low-loss chalcogenide lateral waveguides', IEEE Photonics Technol. Lett., 2001, 13, (3), pp. 182-184

2 Jian, Xu., and Almeida, R.M.: 'Sol-gel derived germanium sulfide planar waveguides', Mater. Sci. Semicond. Process., 2000, 3, pp. 339-344

3 Shaw, L.B., Cole, B., Thielen, P.A., Sanghera, J.S., and Aggarwal, I.D.: 'Mid-wave IR and long-wave IR laser potential of rare-earth doped chalcogenide glass fiber', IEEE J. Quantum Electron., 2001, 37, pp. 1127-1137 